\title{
Core-excitation effects in three-body breakup reactions studied using the Faddeev formalism
}

\author{
A. Deltuva* \\ Institute of Theoretical Physics and Astronomy, Vilnius University, \\ Sauletekio al. 3, LT-10257 Vilnius, Lithuania and \\ Institute for Theoretical Physics II, Ruhr-University Bochum, D-44870 Bochum, Germany
}

(Received 12 December, 2018)

\begin{abstract}
Background: Previous studies of $(d, p)$ reactions in three-body (proton, neutron, nuclear core) systems revealed a nontrivial effect of the core excitation: the transfer cross section cannot be factorized into the spectroscopic factor and the single-particle cross section obtained neglecting the core excitation. This observable, up to a kinematic factor, is the angular distribution of the core nucleus in the $(p, d)$ reaction.

Purpose: The study of the core excitation effect for the most closely related observable in the $(p, p n)$ three-body breakup, i.e., the core angular distribution, is aimed in the present work.

Methods: Breakup of the one-neutron halo nucleus in the collision with the proton is described using threebody Faddeev-type equations extended to include the excitation of the nuclear core. The integral equations for transition operators are solved in the momentum-space partial-wave representation.

Results: Breakup of ${ }^{11}$ Be nucleus as well as of model $A=11 p$-wave nuclei is studied at beam energies of 30,60 , and $200 \mathrm{MeV}$ per nucleon. Angular and momentum distributions for the ${ }^{10} \mathrm{Be}$ core in ground and excited states is calculated. In sharp contrast to $(p, d)$ reactions, the differential cross section in most cases factorizes quite well into the spectroscopic factor and the single-particle cross section.

Conclusions: Due to different reaction mechanisms the core excitation effect in the breakup is very different from transfer reactions. A commonly accepted approach to evaluate the cross section, i.e., the rescaling of singleparticle model results by the corresponding spectroscopic factor, appears to be reliable for breakup though it fails in general for transfer reactions.
\end{abstract}

\section{INTRODUCTION}

Excitation of the core of the nucleus in few-cluster nuclear reactions is expected to be an important dynamic ingredient. Its effect has been studied recently in three-body breakup reactions using the distorted-wave impulse approximation (DWIA) [1, 2] and extended continuum discretized coupled channels (XCDCC) method [3 -5], while for transfer reactions $(d, p)$ and $(p, d)$ exact Faddeev-type equations in the extended Hilbert space have been employed [6]. The latter works demonstrated that, in contrast to a widely accepted assumption, the core excitation (CX) effect in transfer reactions in general cannot be simulated by the spectroscopic factor (SF) only, i.e., by the rescaling of the cross section obtained in the so-called single-particle (SP) model that neglects the CX. The CX effect due to rotational or vibrational quadrupole excitation becomes most evident at energies around $50 \mathrm{MeV}$ in the center-of-mass (c.m.) system, and depends strongly on the angular momentum transfer $\ell$, suppressing the forward-angle differential cross section for $\ell=0$ and enhancing for $\ell=2$ [7]. Since those transfer calculations included breakup to all orders and the breakup operator in the Faddeev formalism is obtained from half-shell elastic and transfer operators, it is interesting and important to investigate the CX effect for $(p, p n)$ breakup reactions. The present work focuses

\footnotetext{
* arnoldas.deltuva@tfai.vu.lt
}

on angular and momentum distributions of the core, the observables that are experimentally measurable but are difficult to achieve the convergence in the XCDCC approach, as found in the previous benchmark comparison of CDCC and Faddeev calculations [8].

Section II presents the three-body Faddeev formalism for breakup reactions including the $\mathrm{CX}$; the unit convention $\hbar=c=1$ is adopted. Analysis of CX effects using several ${ }^{11}$ Be-like model nuclei as examples is given in Sec. III. Section IV contains summary and conclusions.

\section{THEORY}

I consider a three-particle system consisting of a proton $(p)$, neutron $(n)$, and nuclear core $(C)$. Odd-manout notation is used where the channel of a two-particle pair, the third one being a spectator, is labeled according to the spectator and indicated by Greek subscripts. When interacting with nucleons, the core can be excited or deexcited. Ground (g) and excited (x) states are considered simultaneously in the extended Hilbert space [6] whose sectors are coupled by the interaction; the respective components of the operators are indicated by Latin superscripts. Faddeev equations [9] for transition operators $U_{\beta \alpha}$ in the version proposed by Alt, Grassberger, and Sandhas (AGS) [10] were formulated in Ref. [6] for the Hilbert space with several sectors to enable the inclusion 
of the CX, i.e.,

$$
U_{\beta \alpha}^{b a}=\bar{\delta}_{\beta \alpha} \delta_{b a} G_{0}^{a-1}+\sum_{\gamma=p, n, C} \sum_{c=g, x} \bar{\delta}_{\beta \gamma} T_{\gamma}^{b c} G_{0}^{c} U_{\gamma \alpha}^{c a} .
$$

Here $\bar{\delta}_{\beta \alpha}=1-\delta_{\beta \alpha}, E$ is the energy in the c.m. frame, and $G_{0}^{a}=\left(E+i 0-\delta_{a x} \Delta m_{C}-K\right)^{-1}$ is the free resolvent that does not couple Hilbert sectors, but beside the internalmotion kinetic energy operator $K$ contains also the contribution of the excitation energy $\Delta m_{C}$. For each pair $\alpha$ the potential $v_{\alpha}^{b a}$ leads to the respective two-particle transition matrix

$$
T_{\alpha}^{b a}=v_{\alpha}^{b a}+\sum_{c=g, x} v_{\alpha}^{b c} G_{0}^{c} T_{\alpha}^{c a}
$$

to be inserted into three-particle equations (11). Elastic $(\alpha=\beta)$ and transfer $(\alpha \neq \beta=p, n, C)$ operators were calculated in Refs. [6, 7]. In this work I focus on the breakup whose operator is obtained from Eqs. (1) with $\beta=0$, i.e.,

$$
U_{0 \alpha}^{b a}=\delta_{b a} G_{0}^{a-1}+\sum_{\gamma=p, n, C} \sum_{c=g, x} T_{\gamma}^{b c} G_{0}^{c} U_{\gamma \alpha}^{c a} .
$$

Thus, formally it does not require a new solution of integral equations but is given as a quadrature involving elastic and transfer operators $U_{\gamma \alpha}^{c a}$.

The physical breakup amplitudes are determined by the on-shell matrix elements of $U_{0 \alpha}^{b a}$ taken between initial two-cluster and final three-cluster channel states. For a proton impinging with the relative momentum $\mathbf{q}_{p}$ on a one-neutron halo nucleus the initial state $\left|\Phi_{p}\left(\mathbf{q}_{p}\right)\right\rangle=$ $\left|\Phi_{p}^{g}\left(\mathbf{q}_{p}\right)\right\rangle+\left|\Phi_{p}^{x}\left(\mathbf{q}_{p}\right)\right\rangle$ has coupled ground- and excitedstate core components. In the final three-cluster channel $\left|\Phi_{0}^{b}\left(\mathbf{p}_{\beta}^{\prime}, \mathbf{q}_{\beta}^{\prime}\right)\right\rangle$ one can separate ground $(b=g)$ and excited $(b=x)$ states of the core, while the relative pair and spectator momenta $\mathbf{p}_{\beta}^{\prime}$ and $\mathbf{q}_{\beta}^{\prime}$ can be given in any of the three Jacobi sets. Thus, the amplitude for the breakup of a halo nucleus leading to its core state $b$ is given by

$$
\mathcal{T}_{p}^{b}\left(\mathbf{p}_{\beta}^{\prime}, \mathbf{q}_{\beta}^{\prime} ; \mathbf{q}_{p}\right)=\sum_{a=g, x}\left\langle\Phi_{0}^{b}\left(\mathbf{p}_{\beta}^{\prime}, \mathbf{q}_{\beta}^{\prime}\right)\left|U_{0 p}^{b a}\right| \Phi_{p}^{a}\left(\mathbf{q}_{p}\right)\right\rangle .
$$

Starting from the standard expression for the differential three-cluster breakup cross section

$$
\begin{aligned}
d^{6} \sigma= & (2 \pi)^{4} \frac{M_{p}}{q_{p}} \delta\left(E-\frac{p_{\beta}^{\prime 2}}{2 \mu_{\beta}}-\frac{q_{\beta}^{\prime 2}}{2 M_{\beta}}\right) \\
& \times\left|\mathcal{T}_{p}^{b}\left(\mathbf{p}_{\beta}^{\prime}, \mathbf{q}_{\beta}^{\prime} ; \mathbf{q}_{p}\right)\right|^{2} d^{3} \mathbf{p}_{\beta}^{\prime} d^{3} \mathbf{q}_{\beta}^{\prime}
\end{aligned}
$$

the semi-inclusive differential cross section for detecting only the particle $\beta$ is

$$
\frac{d^{3} \sigma}{d^{3} \mathbf{q}_{\beta}^{\prime}}=(2 \pi)^{4} \frac{M_{p}}{q_{p}} \mu_{\beta} p_{\beta}^{\prime} \int d^{2} \hat{\mathbf{p}}_{\beta}^{\prime}\left|\mathcal{T}_{p}^{b}\left(\mathbf{p}_{\beta}^{\prime}, \mathbf{q}_{\beta}^{\prime} ; \mathbf{q}_{p}\right)\right|^{2} .
$$

Here the magnitude of the relative pair momentum $p_{\beta}^{\prime}$ is determined by the energy conservation that is reflected by the $\delta$-function in Eq. (5) with $\mu_{\beta}\left(M_{\beta}\right)$ being the pair (spectator) reduced mass. Further integration of the threefold differential cross section (6) with respect to the magnitude or specific Cartesian components of $\mathbf{q}_{\beta}^{\prime}$ leads to the angular $d^{2} \sigma / d^{2} \hat{\mathbf{q}}_{\beta}^{\prime} \equiv d \sigma / d \Omega_{\beta}$ or momentum $d \sigma / d q_{\beta, i}^{\prime}$ distributions for the particle $\beta$ in the c.m. frame.

The solution of the system of integral equations (1) and the calculation of the quadrature (3) is performed in the momentum-space partial-wave framework, with a subsequent transformation to the plane-wave representation used in Eq. (6). Partial waves with orbital momenta $L_{\alpha}$ up to 5,5 , and 8 for the neutron-core, proton-neutron, and proton-core pair, respectively, are included, the total angular momentum $J$ taking values up to 55 . With these truncations the results for semi-inclusive differential cross sections appear to be converged within $5 \%$, while the difference between the $\mathrm{CX}$ and $\mathrm{SP}$ results, i.e., the CX effect, is converged even better. Thus, the achieved convergence is fully sufficient for a reliable study of the CX effect. Further details on the implementation of threebody reaction calculations including the CX can be found in Refs. [6, 7].

\section{RESULTS}

Previous DWIA [1, 2] and XCDCC [3-5] studies of breakup of one-neutron halo nuclei found an important CX effect for the resonant breakup when the resonance has a significant component with excited core. The present work focuses on the study of the global CX effect in angular and momentum distributions in $(p, p n)$ reactions and its comparison with the CX effect in $(p, d)$ reactions $[\underline{6}, \underline{11}]$. Therefore the example three-body system $p+n+{ }^{10} \mathrm{Be}$ used in this investigation is taken from the corresponding $(d, p)$ study [1]. For the core nucleus ${ }^{10} \mathrm{Be}$ the ground $0^{+}$and excited $2^{+}$states are considered with the excitation energy $\Delta m_{C}=3.368 \mathrm{MeV}$.

The dynamics input for the scattering equations of the previous section is determined by three pairwise multicomponent potentials $v_{\alpha}^{b a}$. For the $n-p$ subsystem there is a number of realistic interaction models such as the charge-dependent Bonn (CD Bonn) potential 12], that will be used also in the present work. This is an important improvement compared to the previous XCDCC calculation [5] of angular and energy distributions that used simple Gaussian $n$ - $p$ potential; the failure of the Gaussian potential in reproducing the breakup results of realistic potentials was demonstrated without CX in Ref. [13]. The neutron-core binding potentials for the spin/parity $j^{\pi}=\frac{1}{2}^{+}$state of ${ }^{11} \mathrm{Be}$ with the neutron separation energy $S_{n}=0.504 \mathrm{MeV}$ is taken over from Ref. [11], whereas Watson [14] and Koning and Delaroche (KD) [15] optical potentials are used for proton-core and neutron-core interaction in remaining partial waves. The proton-core Coulomb interaction is included as well using the method of screening and renormalization [16]. The 


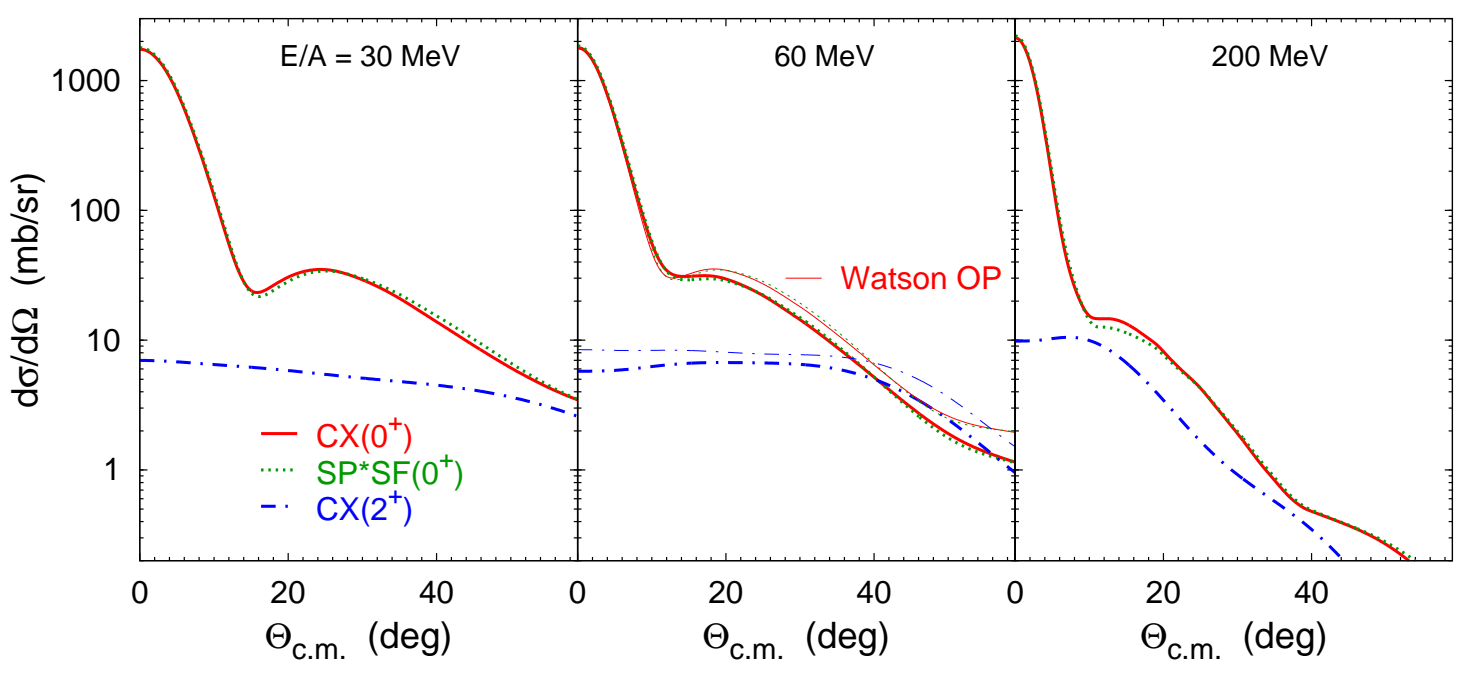

FIG. 1. Semi-inclusive differential cross sections for $\left.{ }^{11} \mathrm{Be}(p, p n)\right)^{10} \mathrm{Be}$ reactions at $E / A=30,60$, and $200 \mathrm{MeV}$ as functions of the ${ }^{10} \mathrm{Be}$ core c.m. scattering angle. Predictions of models with CX, displayed by solid (dashed-dotted) curves for $0^{+}\left(2^{+}\right)$final states of the ${ }^{10} \mathrm{Be}$ core, and without $\mathrm{CX}$, the latter rescaled by $\mathrm{SF}\left(0^{+}\right)=0.854$ and displayed by dotted curves, are compared. In addition to the results based on the KD optical potential, the ones based on the Watson parametrization are shown at 60 $\mathrm{MeV}$ as thin curves.

CX is included via the standard rotational quadrupole deformation of the underlying potentials [17, 18] with the deformation parameter $\beta_{2}=0.67$ and the deformation length $\delta_{2}=1.664 \mathrm{fm}$, while the subtraction technique [19] ensures the two-body on-shell equivalence of the models with and without CX at the given energy. When the reaction is initiated by the beam of $E / A$ energy per nucleon, the energy-dependent optical potential parameters are taken at fixed energy values of $E / A$ and $\frac{1}{2}(E / A)$ for $p-C$ and $n$ - $C$ interactions, respectively. The Watson parametrization was designed for light p-shell nuclei but is constrained by the data up to $50 \mathrm{MeV}$. In contrast, the use of the KD parametrization for ${ }^{10} \mathrm{Be}$ can be criticized as it was fitted to the data for $A \geq 24 \mathrm{nu}-$ clei, but it has an advantage of applicability in a broader energy interval extending up to $200 \mathrm{MeV}$. Comparison of predictions based on Watson and KD potentials will estimate the associated uncertainty.

Previous studies of neutron transfer reactions [6, 7, 11, 19, 20 revealed a nontrivial CX effect in the $(d, p)$ differential cross section. This observable, up to kinematic and spin factors, coincides with the angular distribution of the core in the three-body c.m. frame in $(p, d)$ transfer reactions. It is therefore interesting to investigate the most closely related observable in the $(p, p n)$ breakup, i.e., the angular core cross section $d \sigma / d \Omega_{C}$ in the c.m. frame. The difference to the $(p, d)$ cross section is that the $n$ - $p$ pair is not bound but can be in any continuum state up to the allowed energy; an integration over those states is needed as explained in the previous section. For brevity, the subscript denoting the core in the following will be omitted, both for the angular $d \sigma / d \Omega$ and momentum $d \sigma / d q_{i}$ distributions.
The SP and CX differential cross sections $d \sigma / d \Omega$ as functions of the core c.m. scattering angle $\Theta_{\text {c.m. }}$ at beam energies of 30,60 , and $200 \mathrm{MeV}$ per nucleon are compared in Fig. 1 $E / A=60 \mathrm{MeV}$ roughly corresponds to the maximal CX effect in transfer reactions [11], while $200 \mathrm{MeV}$ should represent the region of relatively high energy with ongoing experimental activities. For a better comparison the SP results are renormalized by the $\mathrm{SF}\left(0^{+}\right)=0.854$ for the ${ }^{10} \mathrm{Be}\left(0^{+}\right)$component in the ${ }^{11} \mathrm{Be}\left(\frac{1}{2}^{+}\right)$bound state. These rescaled SP results simulate well the ${ }^{10} \mathrm{Be}\left(0^{+}\right)$angular distribution in the model including the CX explicitly. Although the shape of the observable changes with the energy, one can see qualitatively the same agreement between these two types of results in all considered cases, also when replacing the $\mathrm{KD}$ optical potential by that of Watson. In fact, the forward peak of the ${ }^{10} \mathrm{Be}\left(0^{+}\right)$angular distribution appears to be quite insensitive to the choice of the optical potential, in contrast to larger angles and the ${ }^{10} \mathrm{Be}\left(2^{+}\right)$angular distribution where the Watson parametrization leads to a larger differential cross section compared to KD, much like for the $(p, d)$ transfer in Ref. [19].

Such a behavior is in sharp contrast with transfer reactions [6, 7, 11, 19, 20] where $d \sigma / d \Omega\left(0^{+}\right)$obtained including the CX cannot be factorized into the SP differential cross section $(d \sigma / d \Omega)_{S P}$ and the associated SF. The size of this CX effect may be characterized by the ratio

$$
R=\frac{d \sigma / d \Omega\left(0^{+}\right)}{\mathrm{SF}\left(0^{+}\right) \cdot(d \sigma / d \Omega)_{S P}}
$$

or its deviation from unity $D=(R-1) \times 100 \%$. In Fig. 2 the latter is compared for breakup and transfer reactions at $E / A=60 \mathrm{MeV}$, i.e., in the energy region showing 


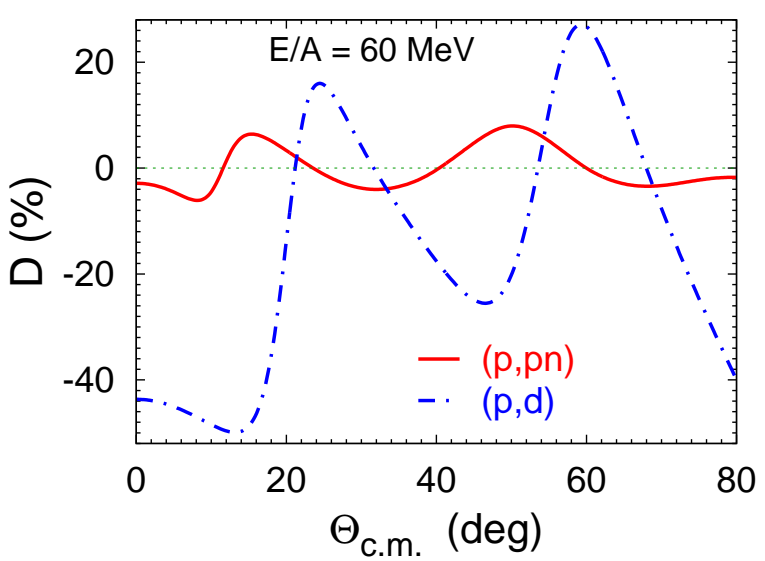

FIG. 2. Core excitation effect for the core angular distribution in breakup (solid curve) and transfer (dashed-dotted curve) reactions, resulting from the proton $-{ }^{11} \mathrm{Be}$ collision at $E / A=$ $60 \mathrm{MeV}$.

the largest CX effect for transfer 11]. Despite some differences in employed optical potentials, the CX effect in the $(p, d)$ reaction shown in Fig. 2 is in agreement with Ref. [1]. It is most sizable at forward angles, where the cross section peaks, reaching almost $50 \%$ in magnitude. On the contrary, the same characteristic quantity $D$ for the breakup stays well below $10 \%$ in magnitude, as can be expected from Fig. 1

The transverse and longitudinal core momentum distributions $d \sigma / d q_{i}$ at the same beam energies are shown in Fig. 3 Again, SP results multiplied by the $\mathrm{SF}\left(0^{+}\right)$ simulate well ${ }^{10} \mathrm{Be}\left(0^{+}\right)$momentum distributions including the CX, with small differences of about $3-4 \%$ seen at the peaks. The optical potential sensitivity is studied at $E / A=60 \mathrm{MeV}$ where the Watson potential predictions are similar to those of $\mathrm{KD}$ for ${ }^{10} \mathrm{Be}\left(0^{+}\right)$at the peaks but are higher by about $20-30 \%$ at the shoulders and for ${ }^{10} \mathrm{Be}\left(2^{+}\right)$momentum distributions. The latter is broader and much smaller in the absolute value, as can be expected due to a small $\mathrm{SF}\left(2^{+}\right)=0.146$, an effectively larger binding $S_{n}+\Delta m_{C}$ and $d$-wave excited core component in ${ }^{11} \mathrm{Be}$.

The study of transfer reactions [19] revealed also the dependence of the CX effect on the internal orbital momentum of the bound state, $p$-wave systems exhibiting weaker CX effect of opposite sign as compared to $s$ wave. For this reason I consider two fictitious $p$-wave model nuclei of mass $A=11$ with the core of ${ }^{10} \mathrm{Be}$ but of spin/parity $\frac{1}{2}^{-}$with neutron separation energies $S_{n}=0.5$ and $5.0 \mathrm{MeV}$. The first value nearly equals the $s$-wave case of the above ${ }^{11} \mathrm{Be}\left(\frac{1}{2}^{+}\right)$breakup study and represents very weakly bound system, while the latter is a more typical value for $p$-shell nuclei. Although both model nuclei differ from the physical ${ }^{11} \mathrm{Be}\left(\frac{1}{2}^{-}\right)$excited state with $S_{n}=0.184 \mathrm{MeV}$, for brevity they will be referred to as ${ }^{11} \mathrm{Be}\left(\frac{1}{2}^{-}\right)$. The parameters of the respec-

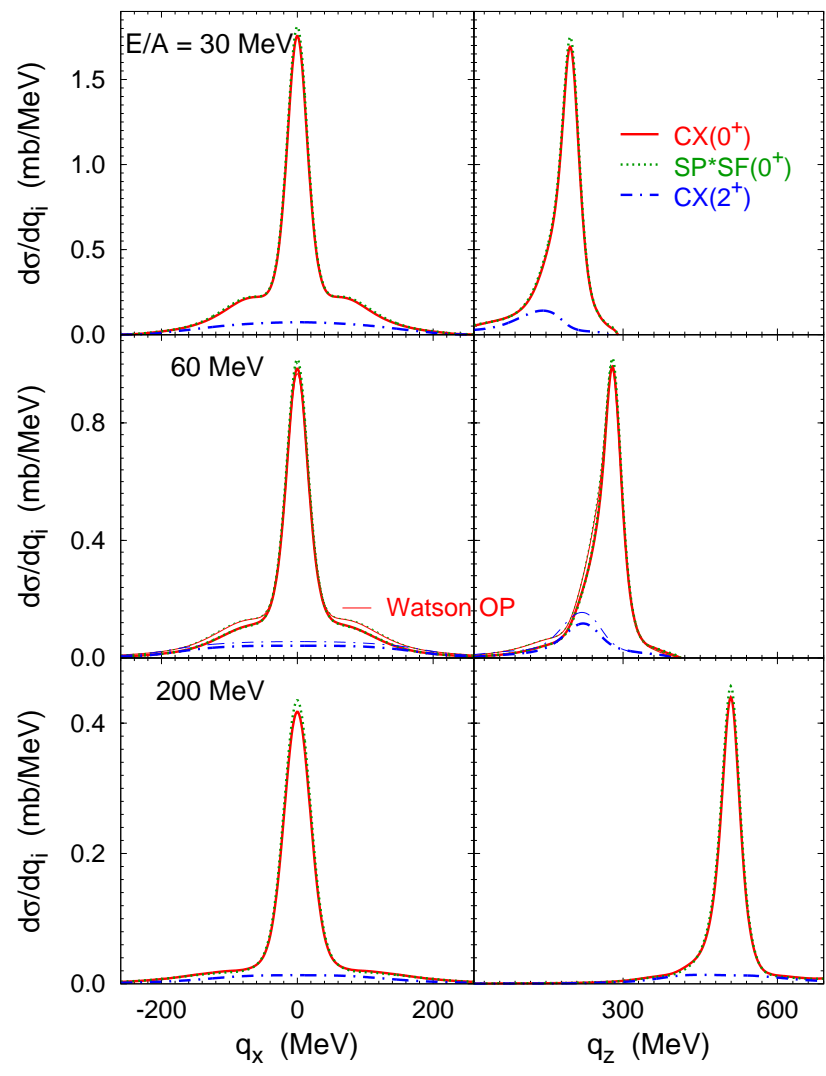

FIG. 3. Transverse (left) and longitudinal (right) core momentum distributions for ${ }^{11} \mathrm{Be}(p, p n){ }^{10} \mathrm{Be}$ reactions at $E / A=$ 30, 60, and $200 \mathrm{MeV}$. Curves are as in Fig. 1.

tive neutron-core binding potentials in the $\frac{1}{2}^{-}$partial wave are taken over from Ref. [11], except for the central Woods-Saxon strength adjusted to the desired binding energy. Otherwise, optical potentials as in the above study of ${ }^{11} \mathrm{Be}\left(\frac{1}{2}^{+}\right)$breakup are used.

The angular distributions of the ${ }^{10} \mathrm{Be}$ core resulting from the $p{ }^{-11} \mathrm{Be}\left(\frac{1}{2}^{-}\right)$breakup at $E / A=60$ and $200 \mathrm{MeV}$ are shown in Fig. 4. Again, SP predictions renormalized by the respective $\mathrm{SF}\left(0^{+}\right)=0.771(0.731)$ for the ${ }^{11} \mathrm{Be}\left(\frac{1}{2}^{-}\right)$binding of 0.5 (5.0) $\mathrm{MeV}$ reproduce reasonably well the observables including CX, except that a more sizable deviation of $20 \%$ is observed at $\Theta_{\text {c.m. }}=0^{\circ}$ for the breakup of the more tightly bound nucleus at the lower beam energy. Such a combination can be associated with larger rescattering contributions, that enhance the importance of nucleon-core interactions and, as a consequence, the CX effect.

Since transverse and longitudinal core momentum distributions show very similar CX effect, I present in Fig. 5 only the former for a more tightly bound model nucleus with $S_{n}=5.0 \mathrm{MeV}$. At a lower beam energy $E / A=60$ $\mathrm{MeV}$ in the peak of the $0^{+}$core transverse momentum distribution there is a clear difference of about $8.5 \%$ between the results including CX and those of the SP model 


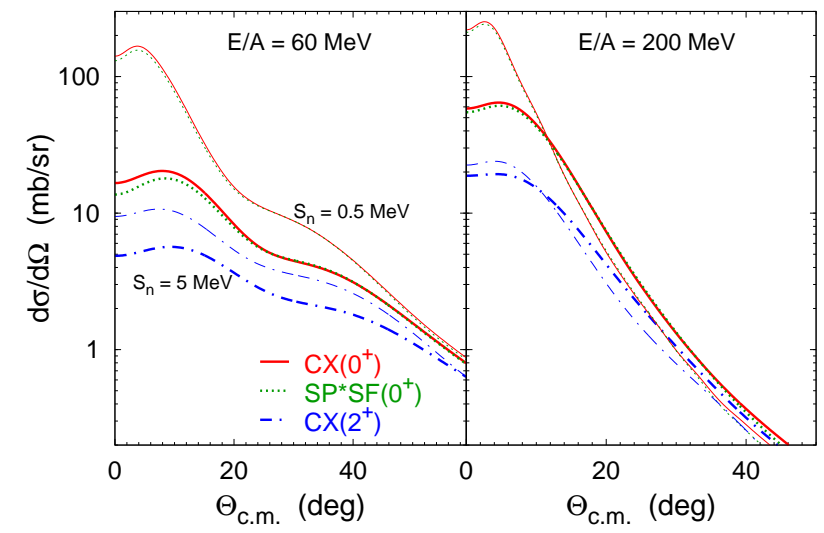

FIG. 4. Semi-inclusive differential cross sections for the breakup of $p$-wave model nuclei as functions of the core c.m. scattering angle at $E / A=60$ and $200 \mathrm{MeV}$. Predictions of models with CX, displayed by solid (dashed-dotted) curves for $0^{+}\left(2^{+}\right)$final states of the core, are compared with rescaled results of the SP model. Thin (thick) curves correspond to the neutron separation energy $S_{n}=0.5 \mathrm{MeV}(5.0 \mathrm{MeV})$ with $\mathrm{SF}\left(0^{+}\right)$being 0.771 (0.731). KD optical potential is used.

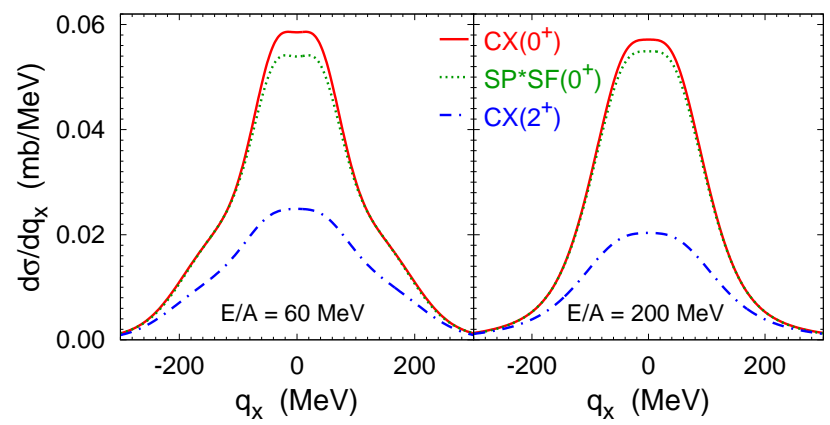

FIG. 5. Transverse core momentum distributions for the breakup of $p$-wave model nucleus with $5.0 \mathrm{MeV}$ binding energy in the collision with proton at $E / A=60$ and $200 \mathrm{MeV}$. Curves are as in Fig. 4.

rescaled by $\operatorname{SF}\left(0^{+}\right)=0.731$. That difference is however reduced to $4.0 \%$ at a higher beam energy $E / A=200$ $\mathrm{MeV}$ where the rescattering is less important. In the case of $S_{n}=0.5 \mathrm{MeV}$, not shown here, the corresponding difference at the peaks of $0^{+}$core momentum distributions amount to $5.9 \%(3.5 \%)$ at $E / A=60 \mathrm{MeV}(200 \mathrm{MeV})$.

Finally, Table【compares also predictions for the integrated three-body breakup cross section $\sigma$ and its components with the core being in a given state. The CX effect for the ground-state core cross section $\sigma\left(0^{+}\right)$is characterized by the parameter $\bar{D}$ defined as $D$ but with the differential cross sections in Eq. (7) replaced by the integrated ones. In all considered cases its magnitude remains below $5 \%$, showing quite weak sensitivity to the beam and binding energies and optical potential. $\bar{D}$ slightly depends on the orbital angular momentum of ${ }^{11} \mathrm{Be}$, being negative for
TABLE I. Integrated three-cluster breakup cross sections (in millibarns) for proton- ${ }^{11} \mathrm{Be}$ collisions at given beam energies (in $\mathrm{MeV}$ ), calculated using various models for ${ }^{11} \mathrm{Be}$, characterized by the neutron separation energy (in $\mathrm{MeV}$ ) and spin/parity. The CX effect for $0^{+}$and total cross sections (in percents) is given in the two last columns. KD optical potential was used, except for the 3rd line results derived from the Watson parametrization.

\begin{tabular}{rrrrrrrr}
\hline \hline$S_{n}\left(j^{\pi}\right)$ & $E / A$ & $\sigma\left(0^{+}\right)$ & $\sigma\left(2^{+}\right)$ & $\sigma$ & $\sigma_{S P}$ & $\bar{D}$ & $\bar{D}_{\Sigma}$ \\
\hline $0.504\left(\frac{1}{2}^{+}\right)$ & 30 & 114.0 & 20.3 & 134.3 & 138.2 & -3.4 & -2.8 \\
$0.504\left(\frac{1}{2}^{+}\right)$ & 60 & 67.1 & 14.9 & 82.0 & 80.4 & -2.3 & 2.0 \\
$0.504\left(\frac{1}{2}^{+}\right)$ & $(\mathrm{W}) 60$ & 72.5 & 20.8 & 93.3 & 87.7 & -3.2 & 6.4 \\
$0.504\left(\frac{1}{2}^{+}\right)$ & 200 & 27.7 & 4.4 & 32.1 & 33.5 & -3.2 & -4.2 \\
$0.500\left(\frac{1}{2}^{-}\right)$ & 60 & 35.9 & 10.3 & 46.2 & 44.5 & 4.6 & 3.8 \\
$0.500\left(\frac{1}{2}^{-}\right)$ & 200 & 19.3 & 5.4 & 24.7 & 24.2 & 3.4 & 2.0 \\
$5.000\left(\frac{1}{2}^{-}\right)$ & 60 & 14.8 & 7.5 & 22.3 & 19.3 & 4.9 & 15.5 \\
$5.000\left(\frac{1}{2}^{-}\right)$ & 200 & 13.6 & 5.9 & 19.5 & 18.0 & 3.4 & 8.3 \\
\hline \hline
\end{tabular}

the breakup of ${ }^{11} \mathrm{Be}\left(\frac{1}{2}^{+}\right)$but positive for ${ }^{11} \mathrm{Be}\left(\frac{1}{2}^{-}\right)$. The CX effect on the total cross section including all states is quantified by $\bar{D}_{\Sigma}=\left(\sigma / \sigma_{S P}-1\right) \times 100 \%$ that is listed in the Table $\prod$ as well. This quantity shows more sensitivity to the optical potential, beam energy, and ${ }^{11} \mathrm{Be}$ bound state properties, mainly caused by the $\sigma\left(2^{+}\right)$contribution.

\section{DISCUSSION AND CONCLUSIONS}

The three-body system of proton, neutron, and nuclear core was treated in the extended Faddeev-type formalism allowing the excitation of the nuclear core. Integral equations for three-body transition operators were solved in the momentum-space partial-wave basis.

Breakup of the one-neutron halo nucleus ${ }^{11} \mathrm{Be}$ in the collision with the proton was considered. Angular and transverse and longitudinal momentum distributions of the ${ }^{10} \mathrm{Be}$ core were calculated at beam energies of 30 , 60 , and $200 \mathrm{MeV} /$ nucleon. For the core detected in its ground state $0^{+}$the results appear to be quite insensitive to the choice of the nucleon-core optical potential. The single-particle calculations that neglect the CX, renormalized by the corresponding spectroscopic factor $\mathrm{SF}\left(0^{+}\right)$of the ${ }^{11} \mathrm{Be}$ bound state, simulate quite well the differential cross sections obtained including the CX.

In order to study the dependence on the internal orbital angular momentum of the bound state, two fictitious ${ }^{11} \mathrm{Be}$-like $p$-wave nuclei with neutron separation energies of 0.5 and $5.0 \mathrm{MeV}$ were considered as well. Despite different shapes of differential cross sections, the CX effect turns out to be quite similar, i.e., the factorization of the cross section for the ${ }^{10} \mathrm{Be}\left(0^{+}\right)$state into the $\mathrm{SP}$ cross section and the respective $\mathrm{SF}\left(0^{+}\right)$remains quite a good approximation. Some deviations are seen mostly at the peaks of distributions, with the most sizable one 
appearing at the lower beam energy and larger binding energy, where one may expect larger nucleon-core rescattering contributions, enhancing also the importance of the CX.

Integrated three-body breakup cross sections were also studied, leading to a similar conclusion — the CX effect for the core ground-state cross section largely consists in the renormalization of the $\mathrm{SP}$ cross section by the corresponding SF. The CX effect on the total cross section depends more strongly on dynamic details.

Thus, CX effects in coupled breakup and neutron transfer reactions turn out to be very different, a probable reason being different reaction mechanisms. Breakup, especially at higher energies, is dominated by the neutronproton quasi free scattering (QFS) where the proton knocks out the neutron from the initial nucleus while subsequent interactions between nucleons and the remaining nuclear core are responsible for the distortion, typically reducing the cross section. Neglecting this distortion, i.e., in the plane-wave impulse approximation, the differential cross section is proportional to the square of the momentum-space bound-state wave function. A consequence of this reaction mechanism is a substantial sen- sitivity to the neutron-core bound-state wave function, surviving also after the distortion. In fact, the differences in Figs. 1 - 5 between $\mathrm{CX}\left(0^{+}\right)$and rescaled SP predictions to some extent may be caused by small differences in the shapes of the corresponding ${ }^{11} \mathrm{Be}$ wave function components. Nevertheless, the similarity of the nuclear wave functions leads to the observed scaling of breakup cross sections calculated with and without the CX. In contrast, the transfer reaction mechanism involves highorder rescattering between all three involved particles, smearing out the sensitivity to the details of the wave function and enhancing the importance of nucleon-core interactions, thereby also the dynamic CX effect.

\section{ACKNOWLEDGMENTS}

I acknowledge the discussions with R. Crespo and A. M. Moro, and the support by the Alexander von Humboldt Foundation under Grant No. LTU-1185721HFST-E and by the Fundação Ciência e Tecnologia (FCT) of Portugal under Contract No. PTD/FISNUC/2240/2014.
[1] A. M. Moro and R. Crespo, Phys. Rev. C 85, 054613 (2012).

[2] A. Moro and J. A. Lay, Phys. Rev. Lett. 109, 232502 (2012).

[3] N. C. Summers and F. M. Nunes, Phys. Rev. C 76, 014611 (2007).

[4] R. de Diego, J. M. Arias, J. A. Lay, and A. M. Moro, Phys. Rev. C 89, 064609 (2014).

[5] R. de Diego, R. Crespo, and A. M. Moro, Phys. Rev. C 95, 044611 (2017).

[6] A. Deltuva, Phys. Rev. C 88, 011601(R) (2013).

[7] A. Deltuva, D. Jurčiukonis, and E. Norvaišas, Phys. Lett. B 769, 202 (2017).

[8] A. Deltuva, A. M. Moro, E. Cravo, F. M. Nunes, and A. C. Fonseca, Phys. Rev. C 76, 064602 (2007).

[9] L. D. Faddeev, Zh. Eksp. Teor. Fiz. 39, 1459 (1960) [Sov. Phys. JETP 12, 1014 (1961)].
[10] E. O. Alt, P. Grassberger, and W. Sandhas, Nucl. Phys. B2, 167 (1967).

[11] A. Deltuva, A. Ross, E. Norvaišas, and F. M. Nunes, Phys. Rev. C 94, 044613 (2016).

[12] R. Machleidt, Phys. Rev. C 63, 024001 (2001).

[13] E. Cravo, R. Crespo, A. M. Moro, and A. Deltuva, Phys. Rev. C 81, 031601(R) (2010).

[14] B. A. Watson, P. P. Singh, and R. E. Segel, Phys. Rev. 182, 977 (1969).

[15] A. J. Koning and J. P. Delaroche, Nucl. Phys. A713, 231 (2003).

[16] E. O. Alt and W. Sandhas, Phys. Rev. C 21, 1733 (1980).

[17] T. Tamura, Rev. Mod. Phys. 37, 679 (1965).

[18] F. Nunes, J. Christley, I. Thompson, R. Johnson, and V. Efros, Nucl. Phys. A 609, 43 (1996).

[19] A. Deltuva, Phys. Rev. C 91, 024607 (2015).

[20] M. Gomez-Ramos and A. M. Moro, Phys. Rev. C 95, 044612 (2017). 